\title{
Heat transfer and air flow near a pair of circular cylinders
}

\author{
Vladimir Mityakov ${ }^{1}$, Vladimir Seroshtanov ${ }^{1, *}$, Alexey Vlasov ${ }^{1}$, Vasily Suchok ${ }^{1}$, Pavel Bobylev ${ }^{1}$ and Nikita Zhidkov ${ }^{1}$ \\ ${ }^{1}$ Peter the Great St. Petersburg Polytechnic University, St. Petersburg, Russian Federation
}

\begin{abstract}
Convective heat transfer is associated with the nature of the flow near the streamlined solid. Since flow properties vary quickly, it is important to fix their momentary values. We propose a technique for studying heat transfer and air flow based on combined use of gradient heat flux measurement and PIV. The paper presents velocity fields near a pair of circular cylinders and distribution of heat flux per unit area on the surface of the second cylinder. Both cylinders were heated with saturated water steam at atmospheric pressure, thereby keeping temperature of the cylinders constant. The experiments were carried out in the range of Reynolds numbers from 480 to 29800. Differences in vortex structure, dead-air region's length etc., and heat transfer are revealed depending on the velocity of free stream and the distance between the cylinders. Use of gradient heat flux sensors allows us to estimate pulsations of heat flux at various points of the second cylinder and compare them with the pictures of instantaneous velocity fields. The results are consistent with data from other authors and show the prospects of the proposed methodology.
\end{abstract}

\section{Introduction}

Flow around pipes is a classic problem of hydrodynamics. Today, there are many experimental and computational papers in which the flow and heat transfer for a single tube and a tube row or bundle of various geometry are studied. So, the authors of [1] managed to obtain distribution of local heat transfer coefficient (HTC) at the surface of a single circular cylinder. They explain the unevenness of HTC value at the surface by the features of flow around bluff solids (presence of laminar-turbulent transition, separated flows, vortexsheet wake formation). In the paper [2], the model of a cylinder of square section is used. Here, the authors have investigated drag coefficient and aerodynamic efficiency. In addition, there are also studied the features related to non-cross flow. In the paper [3], Nakamura et al. determined dependence of the average HTC on the angle between the free stream velocity vector and the cylinder generatrix. This problem is also discussed in [4 $-5]$, where studies were conducted in different regimes.

Today, the issue of heat transfer augmentation during the flow around pipes is also relevant [6].

Since the 1990s, Igarashi has been dealing with this problem, trying to enhance heat transfer using passive (geometric) methods [7-8].

Structure of the vortices behind the cylinder is of particular interest for researchers. These vortices are studied by the SIV [9], PIV [10 - 11], but most often using hot wire anemometers [12 - 14]. The wire with thickness of $10^{-6} \mathrm{~m}$ allows to fix the velocity pulsation almost instantly. Limitations are imposed only by the level of measuring tools existing today.

Based on the foregoing, we summarize: today the combined study of flow and heat transfer in real time is in priority. We also note that despite wide variety of experimental methods, there is a need for a device having high performance while maintaining a satisfactory signal level.

\section{Methods}

The method consists of two modern techniques: a widely used PIV and a unique gradient heat flux measurement. These comprehensive techniques were used in study of air flow and heat transfer near two circular steam heated cylinders installed in the wind tunnel one after the other.

\subsection{Gradient heat flux measurement}

Values of heat flux were determined by gradient heat flux measurement [15]. This method based on the gradient heat flux sensors (GHFSs) with response time of $10^{-8} \ldots 10^{-9} \mathrm{~s}$. GHFSs have been created and integrated into experiment at Peter the Great St. Petersburg Polytechnic University since 1996. Such small response time of GHFSs practically makes them non-inertia instrument for study of most heat transfer types [16 - 18]. Fig. 1 shows the scheme (a) and the general view (b) of the battery GHFS based on bismuth single crystals, which was used in our experiments. The choice of GHFS type was due to experimental conditions (air flow, maximal surface temperature of $100^{\circ} \mathrm{C}$, heat flux per unit area of $0.2 \ldots 5 \mathrm{~kW} / \mathrm{m}^{2}$ ).

The operational principle of GHFS is based on Seebeck's transverse effect. When heat flux passes through a lamel with anisotropy of thermophysical and thermoelectric properties, thermopower arises proportional to heat flux value (Fig. 1). In our

* Corresponding author: vladvik1992@ gmail.com 


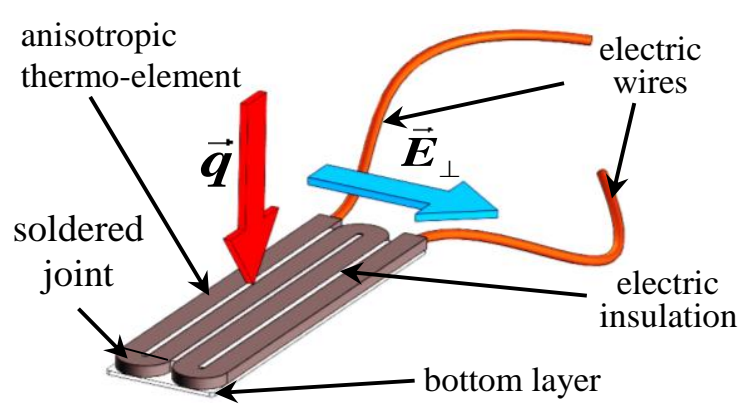

a)

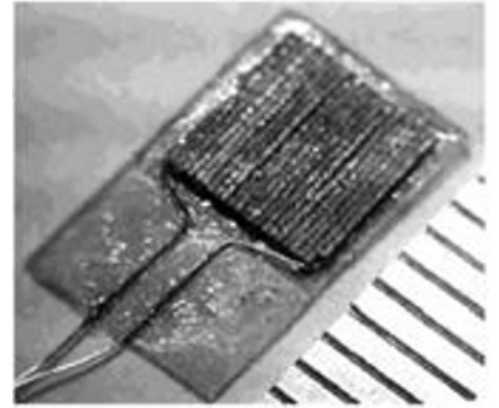

b)

Fig. 1. Scheme and operational principle (a), and general view (b) of the battery GHFS.

experiment the dimensions of GHFSs in plan were of $2 \times 2 \mathrm{~mm}$. Sensors were of $0.2 \mathrm{~mm}$ thick; their volt-watt sensitivity was about $10 \mathrm{mV} / \mathrm{W}$. GHFS were mounted at the second cylinder with a heat conductive compound.

\subsection{PIV}

PIV made us possible to visualize and measure the flow parameters near two cylinders in plane of the laser sheet. PIV works as follows: tracers are fed into the flow: particles are illuminated by laser beam reshaped into the sheet. Supply of tracers was provided by a fog machine. A digital camera fixed the image of tracers at moments of flashes. Our POLIS system [19 - 21] renders possible to adjust the frequency of photographs under the flow velocity. Then, photographs went in the ActualFlow program which calculates the velocity field. Correlation methods of image processing to obtain particle shifts were used.

\subsection{Experimental model}

The experiments were performed in the wind tunnel of Peter the Great Saint-Petersburg Polytechnic University $[22,23]$. The model consists of two circular hollow cylinders mounted on a special frame. The frame allowed to move the second cylinder and thus change the distance $S$ between the cylinders. The model was placed into the Eiffel chamber of the tunnel (Fig. 2).

Both models were heated with the saturated water

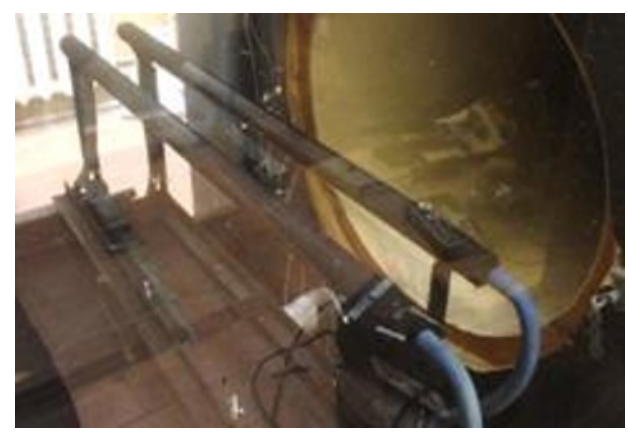

a) steam under the atmospheric pressure; therefore cylinder temperature was close to $100^{\circ} \mathrm{C}$. During the experiment, the second cylinder was rotated around its axis, therefore, the GHFS fixed changes of heat flux in the angle range from frontal to supply point $\left(0 \leq \varphi \leq 180^{\circ}\right)$.

\section{Results and discussion}

Our research was carried out for Reynolds number $\operatorname{Re}=\frac{W \cdot d}{v}=(4 \ldots 2,9) \times 10^{3}$, where $W$ is average air flow velocity, $\mathrm{m} / \mathrm{s} ; d$ is diameter of cylinder, $\mathrm{m} ; \mathrm{v}$ is kinematic viscosity of air, $\mathrm{m}^{2} / \mathrm{s}$. Fig. 5 illustrates the time averaged velocity fields between cylinders for different distances between them. Vectors show the flow direction in plane of light sheet, and colors show the velocity value. The count of photos in our PIV experiments for was of 1000 pairs for each mode.

Visualization results show a stagnant area behind the first cylinder. Also, when the distance between the cylinders increases, this zone lengthens. The distance $S$ also affects the length of the low-energy zone behind the second cylinder: with a smaller distance, it is smaller. For comparison, instantaneous velocity fields in Fig. 4 are presented.

Instantaneous velocity fields, in contrast to the averaged ones, show the presence of velocity irregularities. Locally, the velocity in the wake of the cylinders exceeds the velocity of the oncoming air flow. For high Reynolds numbers, destruction of the vortex

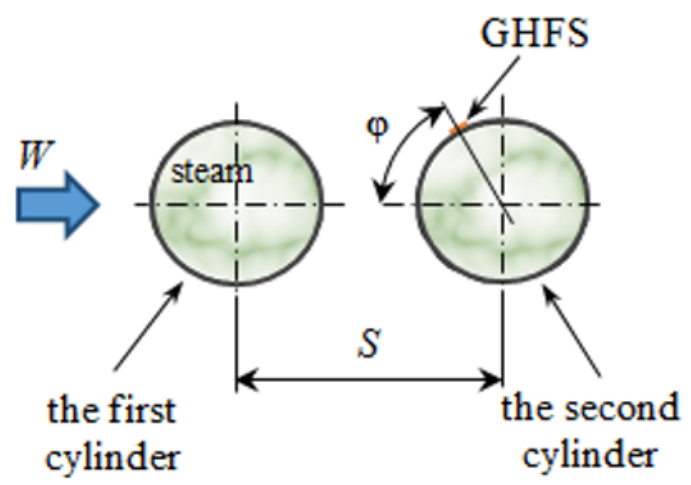

b)

Fig. 2. A pair of cylinders in the Eiffel chamber of the wind tunnel (a), and experimental design (b). 


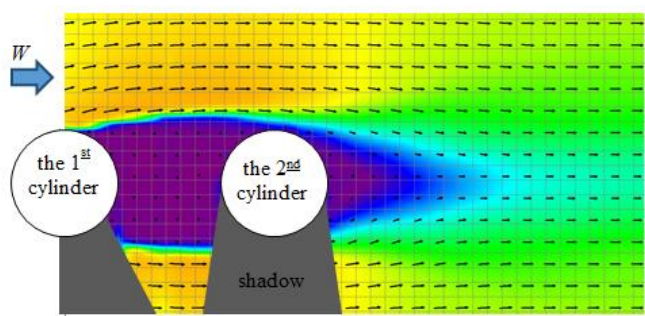

a)

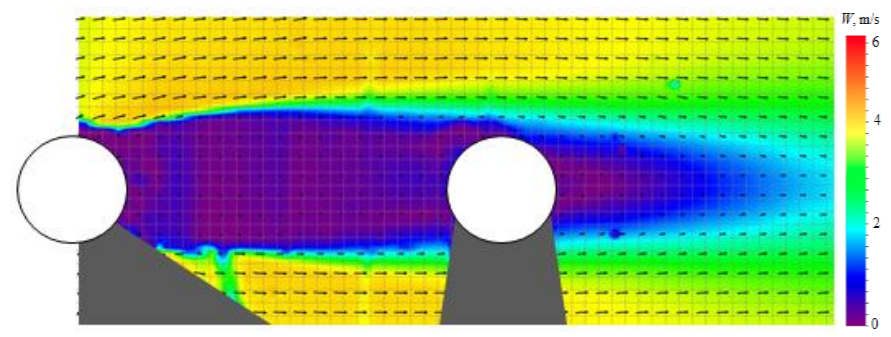

b)

Fig. 3. Time averaged velocity fields near the pair of cylinders for $\operatorname{Re}=4800$ : a) $S=d$, b) $S=3 d$.

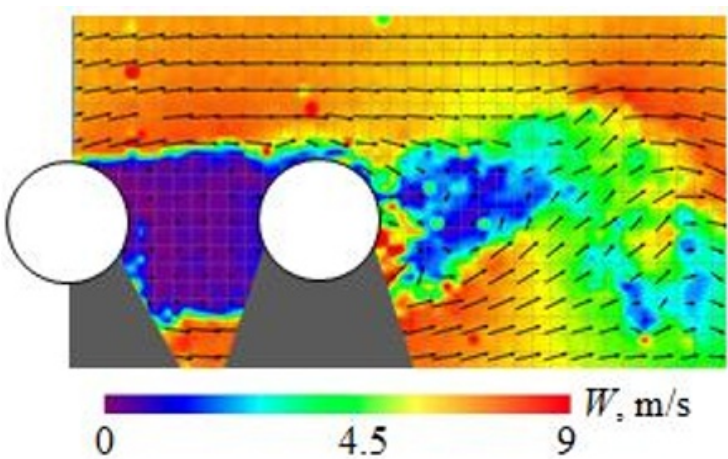

a)

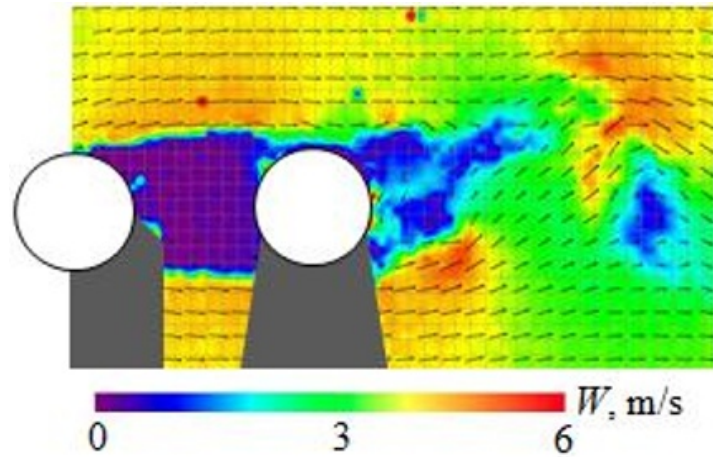

b)

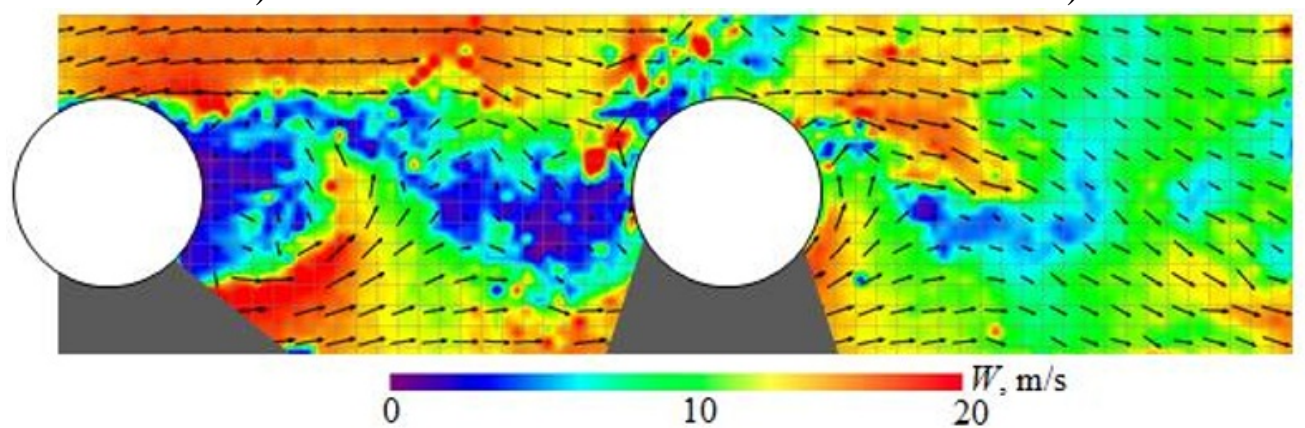

c)

Fig. 4. Instantaneous velocity fields near the pair of cylinders: $a-\operatorname{Re}=9600, S=1 ; b-\operatorname{Re}=4800, S=1 ; c-\operatorname{Re}=29800, S=3$.

descending from the first cylinder is observed.

In Fig. 6 distributions of local HTCs along the surface of the second cylinder are shown.

The graphs show an increase in HTC in the region of $\varphi=30 \ldots 100^{\circ}$ for all regimes at both distances. With increase in the Reynolds numbers, the HTC over the

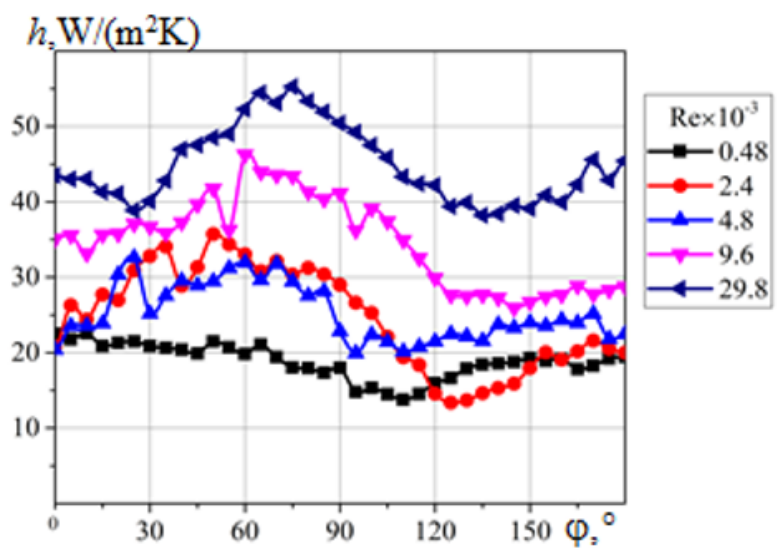

a) entire surface also increases.

Experimental data were processed using the criterion for the intensity of fluctuations of heat flux per unit area. Statistical data processing by standard programs made it possible to obtain in each experiment a set of $n$ values of the average heat flux that can be assumed to be discrete

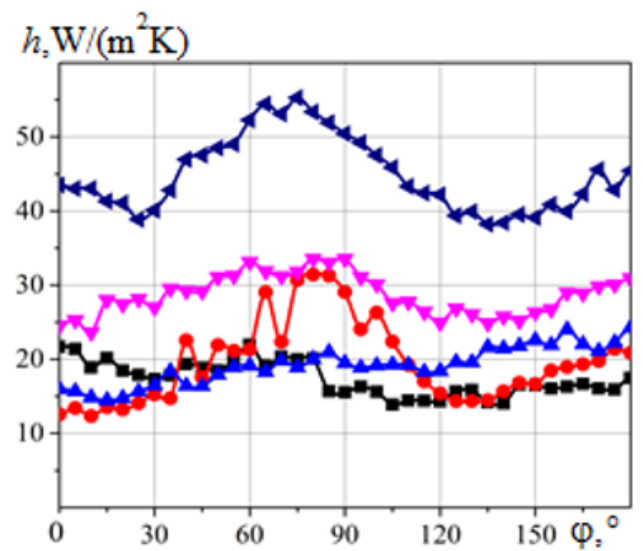

b)

Fig. 5. Dependence of HTC on the angle of rotation $\varphi$ for: $\mathrm{a}-S=d ; \mathrm{b}-S=3 d$. 
and randomly varying quantity (Fig. 5). The required function in this case is represented by the dispersion of heat flux per unit area:

$$
D=\sigma^{2}=\frac{\sum_{i=1}^{n}\left(q_{i}-\bar{q}\right)^{2}}{n-1}, \mathrm{~W}^{2} / \mathrm{m}^{4},
$$

where $\sigma$ is the standard variance and is the arithmetic mean value of the measured local heat flux.

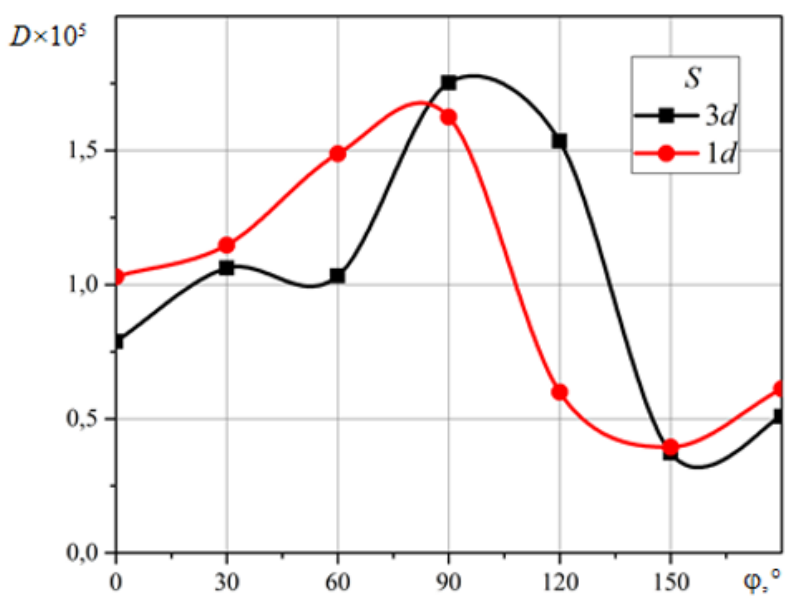

Fig. 6. Dispersion of heat flux per unit area over the second cylinder surface.

It can be seen that individual fluctuation bursts starting from $\varphi=30^{\circ}$. Then, at $\varphi=90 \ldots 100^{\circ}$ fluctuation dramatically decrease. The character of fluctuation essentially escapes from fluctuation on a single cylinder, where they grow with increasing angle of $\varphi$.

\section{Conclusions}

The proposed technique, including superposition of gradient heat flux measurement and PIV allows to study air flow and heat transfer at the surface of the pair of cylinders. Vortexes coming from the first cylinder affect heat transfer at the second one. The appearance and intensity of the vortex track is affected by the distance between the cylinders. Distribution of local HTC for the second cylinder is expectedly dependent on the air flow regime and the distance between the cylinders. The proposed methodology also allows to estimate the level of heat flux per unit area pulsations.

\section{References}

1. Achenbach E. Total and local heat transfer from a smooth circular cylinder in cross-flow at high Reynolds number / Int. J. Heat Mass Transfer. Vol 18, pp. 1387 - 1396, 1975.

2. Bearman P.W. and Harvey J.K. Control of circular cylinder flow by the use of dimples / AIAA Journal Vol. 31, No. 10, 1993, pp. 1753-1756.

3. Giordano R. Vortex shedding in the near wake of a finite cylinder / Giordano R., Astarita T.,
Carlomagno G. M. // 14th IntSymp on Applications of Laser Techniques to Fluid Mechanics, Lisbon, Portugal, 07 - 10 July, 2008.

4. Kaminski D.A., Fu X.D. and Jensen M.K. Numerical and experimental analysis of combined convective and radiative haet transfer in laminar flow over a circular cylinder / Int. J. Heat Mass Transfer. Vol 38, No 17, pp. 3161 - 3169, 1995.

5. Nakamura H., Igarashi T. Unsteady heat transfer from a circular cylinder for Reynolds numbers from 3000 to $15,000 /$ Int. J. of Heat and Fluid Flow 25 (2004) pp. 741-748.

6. Kaikko, J., Mankonen, A., Vakkilainen, E., Sergeev, V.Core-annulus model development and simulation of a CFB boiler furnace (2017) Energy Procedia, 120, pp. 572-579.

7. Ertan Buyruk Heat transfer and flow structures around circular cylinders in cross-flow // Tr. J. of Engineering and Environmental Science 23(1999), $299-315$.

8. E.R. Ranjith et al. / Procedia Technology 24 ( 2016 ) $452-460$

9. Sparrow EM, Gorman JM, Friend KS\& Abraham JP (2013), Flow regime determination for finned heat exchanger surfaces with dim-ples/protrusions. Numerical Heat Transfer, Part A: Applications. 63(4), 245-256.

10. Parnaudeau, P.; Carlier, J.; Heitz, D.; Lamballais, E. Experimental and numerical studies of the flow over a circular cylinder at Reynolds number 3900. Phys. Fluids 2008, 20, 085101.

11. Y. Zhou, H. J. Zhang, and M. Y. Yiu, "The turbulent wake of two side-by-side circular cylinders," J. Fluids Eng.0098-2202 458, 303 (2002).

12. P. Sooraj, Majid Hassan Khan, Atul Sharma and Amit Agrawal, Wake analysis and regimes for flow around three side-by-side cylinders, Experimental Thermal and Fluid Science, 10.1016/j.expthermflusci.2019.02.009, (2019).

13. D. Sumner, Two circular cylinders in cross-flow: A review, Journal of Fluids and Structures, 10.1016/j.jfluidstructs.2010.07.001，26，6，(849899), (2010)

14. Yaryshev N.A. [Theoretical basis of measurement of unsteady temperature] Teoreticheskieosnovyizmereniyanestatsionarnykhte mperatur. Energoatomisdat, Leningrad. Dep-tion, 1990, 254 p. (in Russian).

15. Sapozhnikov SZ, Mityakov VY, Mityakov AV (2013), Gradient heat flux measurement fundamentals. St. Petersburg State Polytechnical University, Saint Petersburg (in Russian).

16. Le-Zakharov, Alexander \& Krivtsov, Anton \& Porubov, A.V.. (2019). Relation between defects and crystalline thermal conduction. Continuum Mechanics and Thermodynamics. 10.1007/s00161019-00807-w.

17. V Yu Mityakov et al 2017 J. Phys.: Conf. Ser. 891 012095

18. Korotkov A, Loboda V, Feldhoff A \&Groeneveld D (2017), Simulation of thermoelectric generators and its results experimental verification. IEEE, Signals, 
Circuits and Systems (ISSCS) International Symposium.

19. Mityakov A, Mityakov V, Sapozhnikov S, Gusakov A, Bashkatov A, Seroshtanov V, Zainullina E \&Babich A (2017), Hydrodynamics and heat transfer of yawed circular cylinder. Int. J. Heat Mass Transf. 115, 333-3

20. Akhmetbekov YK, Bilsky AV, Markovich DM, Maslov AA, Polivanov PA, Tsyryul'nikov IS \&Yaroslavtsev MI (2009), Application of "POLIS" PIV system for measurement of velocity fields in a supersonic flow of the wind tunnels. Thermophysics and Aeromechanics 16(3), 325333.V Yu Mityakov et al 2017 J. Phys.: Conf. Ser. 891012095

21. Gusakov AA, Kosolapov AS, Markovich DM, Mityakov AV, Mityakov VY, Mozhayskiy SA \&Nebuchinov AS \&Sapozhnikov SZ (2014), Simultaneous PIV and gradient heat flux measurement of a circular cylinder in cross-flow. Applied Mechanics and Materials 629, 444-449.

22. website: www.https://www.spbstu.ru/

23. Zasimova, Marina \& Ivanov, Nikolay \& Ris, Vladimir \& Tschur, N.. (2018). Heat Transfer in a Staggered Bare-Tube Bank Immersed in a Vast Water Pool. High Temperature. 56. 711-718. 10.1134/S0018151X18040211. 